\title{
Pathogenesis of Severe Acute Respiratory Syndrome Coronavirus-2 (SARS-CoV-2) Respiratory Infection
}

\author{
Abolaji Samson Olagunju ${ }^{1 *}$, Ifeoluwa Peace Oladapo², Samson Oluwapelumi Kosemani ${ }^{1}$, Folashade \\ Gloria Olorunfemi ${ }^{3}$, Adesewa Sukurat Adeyemo²
}

${ }^{1}$ Department of Biochemistry, University of Ibadan, Ibadan, Nigeria

${ }^{2}$ Department of Zoology, University of Ibadan, Ibadan, Nigeria

${ }^{3}$ Department of Biochemistry, University of Lagos, Lagos, Nigeria

Corresponding Author: Abolaji Samson Olagunju, MSc Student, Department of Biochemistry, University of Ibadan, Ibadan, Nigeria. Tel: +234-7068-528-329, Email: abolajisamson05@gmail.com

Received June 7, 2020; Accepted July 15, 2020; Online Published July 25, 2020

\begin{abstract}
With the occurrence of a mysterious pneumonia in the Hubei province (Wuhan) of China in December 2019, a different coronavirus, the severe acute respiratory syndrome coronavirus 2 (SARS-CoV-2), has commanded global awareness and has been named by the World Health Organization (WHO) as a public health emergency of international concern. Two other coronavirus infections (SARS and MERS) were also characterized by severe respiratory distress in 2002-2003. In addition to the new coronavirus, the emerging infectious diseases resulting in universal spread are caused by the $\beta$-coronavirus strains. Even though coronaviruses typically target the upper and/or lower respiratory tract, viral shedding into the plasma or serum is frequent, and the human coronavirus (CoV) represents $15 \%-30 \%$ of respiratory syndromes, including common colds. Based on a recent hypothesis, SARS-CoV-2 has been shown to induce lung injury by inhibiting the angiotensin converting enzyme-2 (ACE-2) and could possibly attack organs with high expression. With the lack of a vaccine or major treatment for the disease, palliative care is provided for individuals already infected with the virus. The aim of this review is to discuss the influence and relationship of the coronavirus, particularly SARS-CoV-2, on the respiratory system with a proposed mechanism of action in lung injury and pathogenesis.

Keywords: COVID-19, Angiotensin Converting Enzyme 2, Severe Acute Respiratory Syndrome Coronavirus-2, Communicable Diseases, Public Health, Lung Injury
\end{abstract}

Citation: Olagunju AS, Oladapo IP, Kosemani SO, Olorunfemi FG, Adeyemo AS. Pathogenesis of severe acute respiratory syndrome coronavirus-2 (SARS-CoV-2) respiratory infection. Int J Travel Med Glob Health. 2020;8(4):137-145. doi:10.34172/ijtmgh.2020.24.

\section{Introduction}

Coronavirus disease 2019 (COVID-19) is an acute respiratory disease which can cause respiratory breakdown and even death. It is caused by the novel severe acute respiratory syndrome coronavirus 2 (SARS-CoV-2). Prior epidemics of previously known coronavirus diseases, severe acute respiratory syndrome (SARS) and Middle East respiratory syndrome (MERS), were linked with similar clinical features and effects. ${ }^{1}$ Coronaviruses are enclosed, positive-sense, single-stranded RNA viruses. A research done by Lamarre and Talbot $^{2}$ showed that the virus is susceptible to acidity, alkalinity, and heat and can survive at $4^{\circ} \mathrm{C}$. There was no substantial decrease in the infectious titre after 25 cycles of thawing or freezing. It might be of a general opinion that patients who are asthmatic or have chronic obstructive pulmonary disease (COPD) are more susceptible to SARSCoV-2 (COVID-19) infection. Nevertheless, both diseases are under-represented in the comorbidities described among individuals with COVID-19 in contrast with the overall burden of disease in the general population. In contrast, there is a high occurrence of diabetes among SARS or COVID-19 patients. The lung is the weakest internal organ prone to infection due to its continual contact with airborne particles. Not less than 2 billion people are exposed to poisonous smoke, 1 billion individuals breathe in adulterated open-air, and 1 billion inhale tobacco smoke worldwide.

Respiratory injury causes disability and death to every social class and imposes a universal health problem in all regions of the world. Five of these respiratory diseases are among the most common causes of severe illness and death globally, namely lung cancer, tuberculosis, COPD, acute lower respiratory tract infection, and asthma. ${ }^{3}$

In total, over 1 billion people suffer from either severe or long-lasting respiratory illnesses with about four million deaths annually. ${ }^{4}$ Newborns and young children are more disposed to respiratory diseases which lead to the death of a

Copyright $\odot 2020$ The Author(s). This is an open-access article distributed under the terms of the Creative Commons Attribution License (http:// creativecommons.org/licenses/by/4.0), which permits unrestricted use, distribution, and reproduction in any medium, provided the original work is properly cited. 
total of 9 million children below the age of 5 .

\section{Coronavirus Diversity}

Coronaviruses are one of the largest known RNA, separated into 4 genera: $\alpha$-CoVs, $\beta$-CoVs, $\gamma$-CoVs, and $\delta$-CoVs (Figure 1) ${ }^{5}$ : $\alpha-\mathrm{CoVs}$ and $\beta-\mathrm{CoVs}$ infect warm-blooded animals, while $\gamma$-CoVs and $\delta$-CoVs infect birds as well as mammals. ${ }^{6}$ To date, seven coronaviruses have been found to infect humans and cause respiratory diseases, of which four are common human CoVs (HCoVs) which lead to selflimited upper respiratory infection: $\mathrm{HCoV}-\mathrm{OC} 43, \mathrm{HCoV}-$ 229E, HCoV-HKU1, and HCoV-NL63.

SARS-CoV-2 presents milder clinical symptoms than SARS and MERS according to existing reports. ${ }^{9}$ In 2019, Zhu et $\mathrm{al}^{10}$ identified SARS-CoV-2 as a new member of the $\beta$-CoVs, isolated it from human airway epithelial cells, and categorized it by next generation sequencing (NGS) in January 2020.

\section{SARS-CoV-2 (COVID-19)}

An epidemic of an unidentified pneumonia started in Wuhan, China in December 2019. Most index cases were linked to a particular seafood market. ${ }^{10}$ Following this, researchers isolated and identified a new beta-coronavirus; the genome has $86.9 \%$ homology with an already known bat SARS-like coronavirus genome (bat-SL-CoVZC45, MG772933.1) and varies distinctively from human SARS-CoV and MERS-CoV. ${ }^{11}$ Patients with COVID-19 generally have malaise and lower respiratory tract symptoms. Limited data indicates that viral RNA could be discovered in the plasma or serum of patients with COVID-19. Viremia was detected in approximately $15 \%$ of the first 41 COVID-19 patients in Wuhan. There was a low RNA concentration with no disparity found between patients in intensive care units and those with mild symptoms as a result of the average polymerase chain reaction (PCR) cycle threshold value, which was 35.1 (95\% CI: 34.7-35.1). One of the 41 individuals was positive for SARS-CoV-2 RNA without symptoms of fever. ${ }^{9}$ Research carried out on a family of 6 revealed that serum from 1 of the 6 family members revealed a weak positive result for SARS-CoV-2 RNA and a 10-year-old child was confirmed as an asymptomatic carrier. ${ }^{12}$ The virus being cosmopolitan, reports from Vietnam, ${ }^{13}$ Germany, ${ }^{14}$ and the United States ${ }^{15}$ have demonstrated the clinical symptoms, diagnosis, and treatment of the new coronavirus disease (COVID-19). A report described transmission by contact with an asymptomatic carrier in Germany where a Chinese national attended business meeting in Germany and infected not less than 2 business partners during the incubation period. ${ }^{13}$ This report shows that compared to SARS patients, COVID-19 individuals might be infectious during an asymptomatic incubation period. However, the investigators did not interrogate the Chinese traveler who was later found to have been symptomatic at the time of the direct contact. ${ }^{13}$

\section{Morphology, Structure, and Replication of Severe Acute Respiratory Syndrome Coronavirus}

The virion of SARS-CoV is globular with a diameter of 78 $\mathrm{nm}$, nucleocapsid, and encapsulated in the peplomers, ${ }^{16}$ which gives it the typical crown-like appearance. The virus

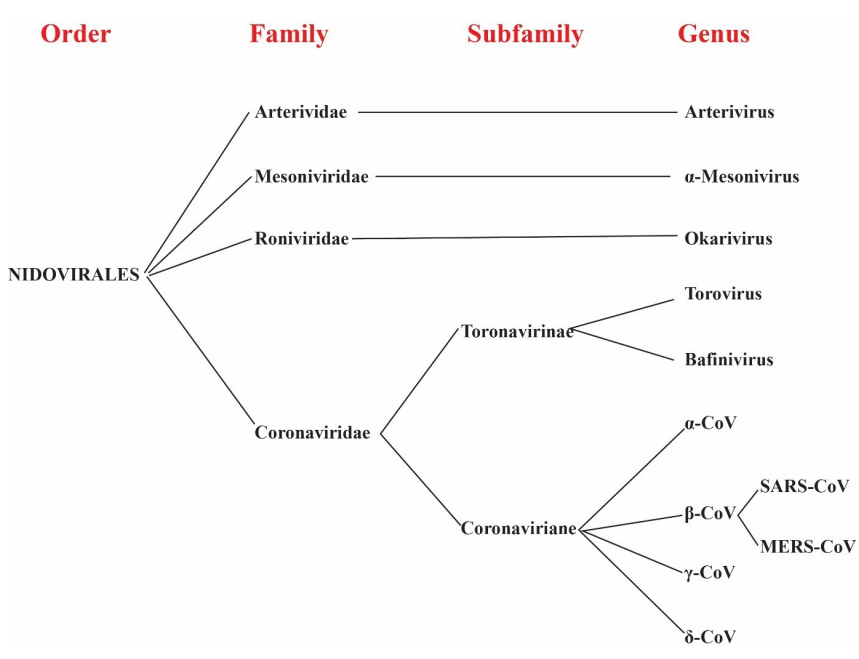

Figure 1. Classification of Coronaviruses., ${ }^{7,8}$

gains entrance by attaching itself to the host cell receptor angiotensin converting enzyme-2 (ACE-2). ${ }^{17}$ Polyanion compounds inhibit the access of SARS-CoV to target cells. This shows that SARS-coronavirus-encapsulated proteins may be positively charged and interact with heparin sulfate proteoglycans which are negatively charged on the surface of target cells. ${ }^{18}$ Coronaviruses replicate in the cytoplasm where viral RNA is synthesized. ${ }^{19}$ These changes comprise doublemembrane vesicle formation, granulations in the cytoplasm, and the presence of nucleocapsid inclusions. Replicase, a viral RNA polymerase, is the first gene to be translated. It initially transcribes full-length, anti-sense, or negative strand copies of the genome. The anti-sense strands are at this point used as a template to generate mRNAs that transcribe viral genes. The subgenomic transcripts are nested, having similar 5' regions, non-translated, and a 3' poly-A tail. Diverse nested transcripts are then produced by the activity of the viral RNA polymerase and not by splicing. Then, there is an interaction between the viral RNA polymerase and a repeated intergenic sequence (TRS-transcription regulating sequence) located between the viral genes. This interaction permits the connection between the 5 ' leader sequence and the beginning of each gene.

Rough areas comprising viral RNA, proteins absent in cells invaded by other coronaviruses, could be present in cells infested by the SARS-CoV, making the section a viral translation center. ${ }^{20}$ Viral particles are found in the Golgi body and accumulate in dilated vesicles which are then conveyed to the surface of the cell where they are released via exocytosis. There is a different biological characteristic between SARS$\mathrm{CoV}$ and formerly identified coronaviruses.

SARS-CoVs are tropic for Vero cells (a cell-line acquired from the epithelial cells of the kidney of African green monkeys). Unlike other coronaviruses which can develop at a low temperature and cause respiratory tract diseases, SARS$\mathrm{CoV}$ grows at $37^{\circ} \mathrm{C} .{ }^{18}$ The SARS-CoV genome is between 29705 and 29751 nucleotides (NCBI Sequence Viewer). The SARS virus genome differs from other formerly recognized groups of coronaviruses and possesses a weak antigenic connection to other coronaviruses (OC43 and 229E).

The SARS coronavirus lacks hemagglutinin-esterase 
which can be found in some group 2 and 3 coronaviruses, but it possesses one single proteinase (papain-like) which is present in group 3 coronaviruses. ${ }^{21}$ This led to the proposal of a new coronavirus group (group 4), consisting of the SARS coronavirus as the lone member. The discovery of the SARS coronavirus initiated the search for other, formerly unidentified human coronaviruses. The organization of SARS coronavirus is related to other coronaviruses with the gene order of 5', replicase [rep], spike [S], envelope [E], membrane $[\mathrm{M}]$, nucleocapsid $[\mathrm{N}], 3$, flanked by short untranslated regions..$^{20,22}$ Sequences possibly encoding five additional non-structural proteins are interspersed between the open reading frames (ORFs) and $\mathrm{N}$. The SARS-CoV genome comprises 11 predicted ORFs that can encode as many as 23 mature proteins. ${ }^{23}$ The two major ORFs which occupy about two-thirds of the genome are coded as ORFla and $b$. Through proteolysis, the polyproteins are cleaved to form non-structural proteins that are the RNA-dependent RNA polymerase (Rep) and an ATPase helicase (Hel) (Figure 2).

The SARS coronavirus possesses some genetic features that differ slightly from other coronaviruses, comprising a short anchor in the S protein, and it differs in number and location of ORF, presence of a single PLP-protease, and a sole, short, lysine-rich region which is present in the nucleocapsid protein. The biological significance of these variations is still unknown. ${ }^{24}$

\section{Clinical Manifestation and Pathophysiology}

To report the pathogenetic mode of SARS-CoV-2, it is vital to consider its viral structure and genome. Coronaviruses are known to have the biggest recognized RNA genomes, $30-32 \mathrm{~kb}$ with a 50-cap structure and 30-poly-A tail. The transcription works through the replication-transcription complex (RCT). At the transcription regulatory sequences, which are situated in the middle of the transcription ORFs, termination occurs. ${ }^{25}$

At least 6 ORFs can be present in the atypical CoV genome. Among these, a frame-shift between ORF1a and b directs the formation of either ppla and b polypeptides, which are processed by virally encoded main protease (Mpro) or chymotrypsin-like protease (3CLpro). ${ }^{25}$ Other ORFs encode for structural proteins, such as envelope, membrane, spike, and accessory protein chains and nucleocapsid proteins. ${ }^{25,26}$

There is an association between the pathophysiology and virulence mechanisms of coronaviruses, and SARS coronavirus and the structural and non-structural proteins. Various studies emphasize that non-structural proteins (nsps) can stop the host innate immune response. ${ }^{27}$ Virus pathogenicity is facilitated by the envelope as it enhances assembly and release of the virus. However, the pathogenic mechanisms that lead to pneumonia seem complex..$^{25-27}$ The existing research figures show that the viral infection has the ability to produce an extreme immune reaction in the host, referred to as a cytokine storm, which leads to tissue damage. During a cytokine storm, interleukin-6 (IL-6) is synthesized by the activation of leukocytes, which then interact with a large number of tissues and cells. ${ }^{28} \mathrm{IL}-6$ is a pro-inflammatory cytokine which can have an anti-inflammatory effect. It rises during autoimmune disorders, inflammatory diseases, cardiovascular diseases, some types of cancer, and infections. ${ }^{29}$ It can stimulate some growth cells and B lymphocytes differentiation and can prevent the growth of other cells. This cytokine plays a vital role in stimulating the production of acute phase proteins, thermoregulation, bone maintenance, and central nervous system function. ${ }^{30,31}$ It is also involved in the cytokine release syndrome pathogenesis, a severe systemic inflammatory syndrome characterized by multiple organ dysfunctions and fever. The virus passes through the mucous membrane, mainly the nasal and laryngeal mucosa, passes through the respiratory tract, and enters into the lungs. The virus then attacks the targeting organs expressing ACE2, for example the lungs, gastrointestinal tract, renal system, and heart. ${ }^{29-31}$ There is a second attack of the virus which aggravates the patient's condition 7 to 14 days after onset. B lymphocytes can decrease at the early phase of the malady, affecting the production of antibodies.

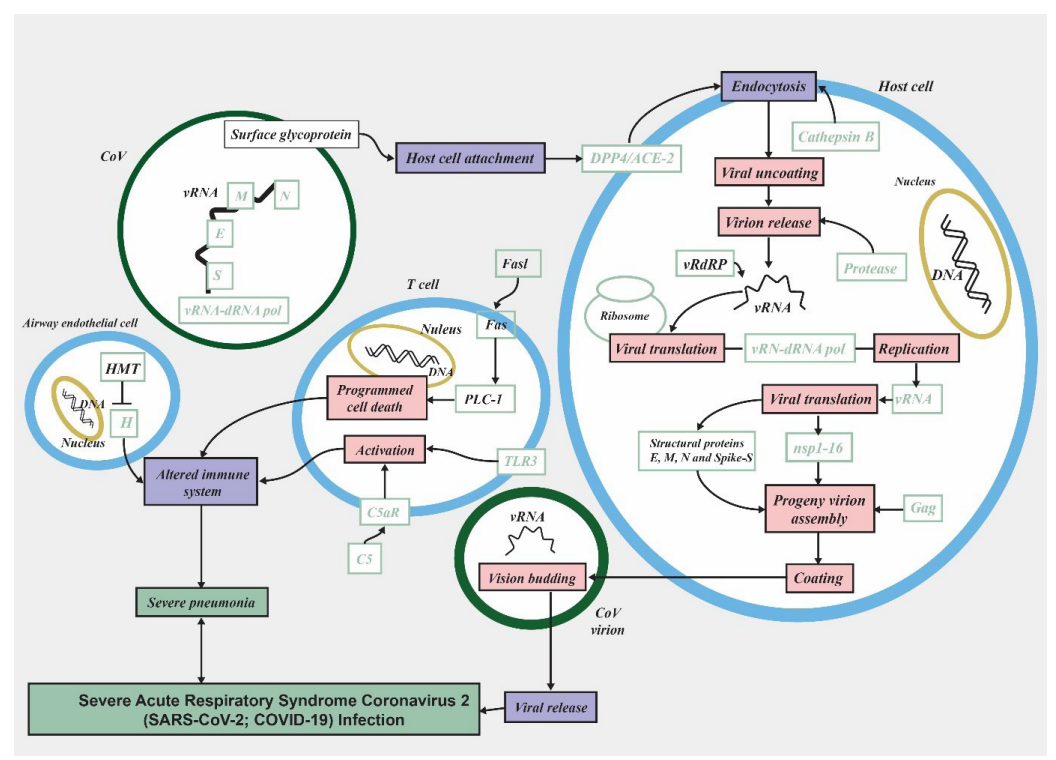

Figure 2. Targetscape of COVID-19 Infection. ${ }^{32}$ 


\section{Viral Invasion and Respiratory Disease}

Respiratory disease such as the common cold is a commonly known short illness. The major clinical manifestations include upper respiratory tract and nasal symptoms (Figure 3). Understanding the mechanism of interaction between the virus and the epithelium is crucial ${ }^{33}$ during viral infection. While some infected individuals suffer nasal congestion, others may have no certain symptoms. The reason remains unclear. Human coronaviruses $(\mathrm{H}-\mathrm{CoV})$ are the second most widespread cause of the common cold accounting for $15 \%$ to $30 \%$ of confirmed viral infections and may lead to lower respiratory diseases like asthma, ${ }^{34}$ even though the prevalence of asthma in COVID-19 positive patients is low, and this might be due to one or more factors.

Chronic respiratory disease could be under-diagnosed in individuals with SARS-CoV-2 in contrast to diabetes, particularly in China. This appears unlikely, as the results reported from Italy on March 23, 2020 state that among 355 (mean age $=79.5$ years) infected patients, $20.3 \%$ had diabetes, while COPD was not listed as a co-morbidity for any individual. However, the data reported from the United States on March 31, 2020 showed that $8.5 \%$ of infected patients had chronic respiratory disease, while $10.2 \%$ had diabetes. Global burden of disease figures for the entire populace showed that $11.3 \%$ had chronic respiratory diseases and $10.2 \%$ had diabetes; this data is centered on only 7162 of the 74,439 individuals reported. ${ }^{35}$ Another reason could be the fact that chronic respiratory disease may protect against SARS-CoV-2, probably through a diverse immune response stimulated by the chronic disease itself. Meanwhile, this concept does not correlate with the observation that among COVID-19 patients who have COPD as a co-morbidity, mortality is increased, as otherwise would be expected. ${ }^{35,36}$ Also, treatments used by individuals with chronic respiratory disease can decrease the threat of infection. It is vital to note that about half of the individuals who have chronic obstructive pulmonary disease in China take medications that are standard, but approximately $75 \%$ of people in China with asthma make use of inhaled corticosteroids. ${ }^{37}$ Moreover, in in-vitro models, the use of inhaled corticosteroids alone or in combination with bronchodilators have shown to inhibit cytokine production and replication of the coronavirus. ${ }^{38,39}$

Low quality proof also exists from a series of cases in Japan, in which there was an improvement in three COVID-19 individuals who required oxygen but not ventilation after using inhaled ciclesonide. ${ }^{40}$ However, a control group was not used, and whether there will be rapid improvement in these individuals cannot be ascertained. However, the probability that inhaled corticosteroids could inhibit the progress of symptomatic infection or severe presentations of SARS-CoV-2 cannot be ignored. Comparatively, a systematic assessment on systemic corticosteroid usage to treat SARS, once confirmed, presented no benefit but potential harm. ${ }^{41}$

\section{Respiratory Diseases}

The respiratory system is made up of organs grouped into upper respiratory tract (pharynx, larynx, trachea, and nasal cavity) and lower airways (lungs, bronchi, and bronchioles). ${ }^{42}$ Epithelial cells which comprise an active physical blockage against disease-causing organisms' are a vital aspect of the innate immune system that covers the inner part of these organs. Another structure of the respiratory membrane is the mucociliary structure, located in the nasal cavity close to the distal areas of the lungs, which consists of a layer of mucus formed by goblet cells which retain a continual flow via the ciliary movement in the luminal surface respiratory epithelium. The lung lacks these structures but has alveolar macrophages which are responsible for removing pathogens. This structure provides protection against respiratory viral infections. ${ }^{43}$ Regardless of these protective mechanisms, the host's respiratory system can still be infected by the virus by attaching to particular receptors existing on the mucosa epithelial cells, thus evading its elimination by the mucociliary system or phagocytes. ${ }^{44}$ The respiratory tract is the entry channel for many viruses that infect humans. ${ }^{45}$

Large particles are typically confined in the sinuses and the turbinates which can eventually affect upper respiratory infections, while smaller particles can spread to the alveolar spaces and eventually lead to lower respiratory tract infections. ${ }^{46,47}$

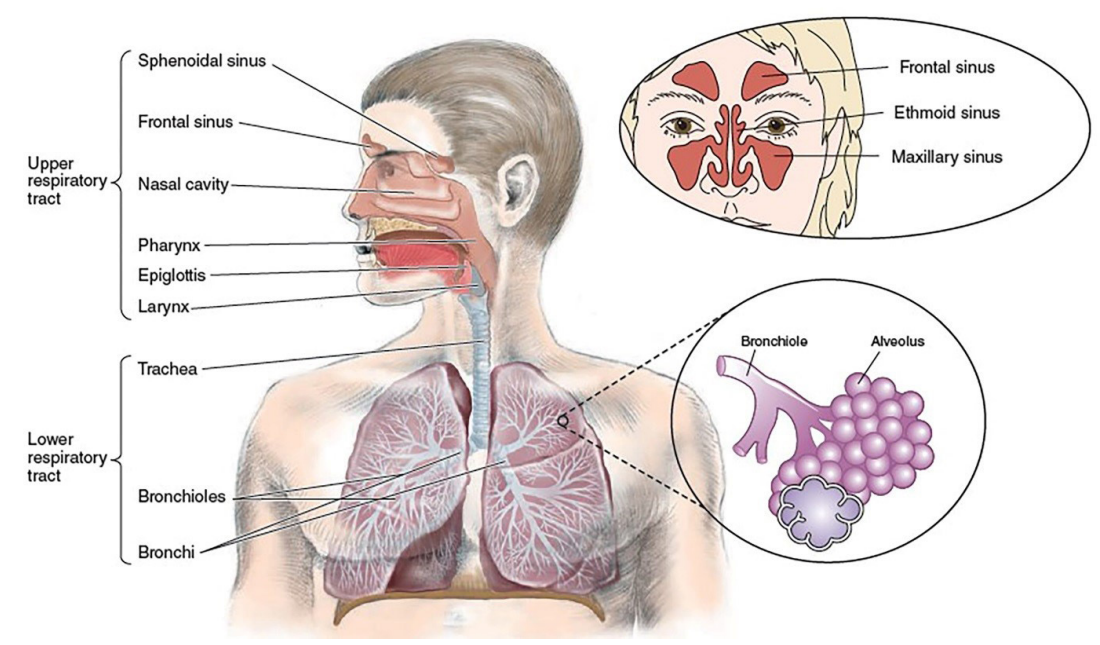

Figure 3. Anatomy of the Respiratory System. ${ }^{48}$ 
Chronic respiratory diseases are diseases of the airways and other structures of the lung. They include a variety of chronic respiratory ailments such as asthma, COPD, interstitial lung disease, occupational lung diseases, and others.

Viruses that cause respiratory disease in both the upper and lower airways are grouped in different families: Coronaviridae Orthomyxoviridae, Picornaviridae, Paramyxoviridae Adenoviridae, Reoviridae, and Herpeviridae (Figure 4). Once the virus penetrates through, infections occur; for example, coronavirus, influenza, parainfluenza virus, rhinovirus, bocavirus, respiratory syncytial virus, and meta-pneumovirus seldom cause lower respiratory infections. Other viruses, for example measles, mumps, rubella, varicella, and herpes among others, pass through the airways and then travel down to other organs.

\section{Viral Invasion of the Upper Respiratory Tract}

In humans, upper respiratory infections are major, with a higher incidence in the winter and lower incidence in the summer. They are also the major cause of medical consultation leading to frequent work and school absenteeism. Examples of viruses associated with infections of the upper respiratory tract include coronavirus $(\mathrm{CoV})$, rhinovirus $(\mathrm{RV})$, parainfluenza (PIV), adenovirus (AD), influenza A (IA), respiratory syncytial virus (RSV), human meta-pneumovirus (hMPV), and human bocavirus ( $\mathrm{HBoV})$ with variable clinical manifestations. All individuals, particularly children, are susceptible to infection by these viruses. Major syndromes in the upper airway include pharyngitis, adenoiditis, sinusitis, croup, laryngitis, and nasopharyngitis ${ }^{48}$ (Figure 4).

\section{Viral Invasion of the Lower Respiratory Tract}

Infections of the lower respiratory tract by viruses have only a small percentage, but a high death rate with children being the most susceptible, even though adults can also be infected. The malady is increased by numerous factors such as metabolic disorders, anatomical, immunological, or other diseases, including COPD, asthma, or AIDS. Viral infection may damage the respiratory epithelium without obvious clinical symptoms; however, it may have implications in lung disease, such as in chronic suppurative disease which reduces lung function significantly after asymptomatic and symptomatic viral infections (Figure 5). ${ }^{49}$

Proposed Mechanism of Lung Injury in COVID-19 Patient In the host, the life cycle of the novel coronavirus consists of five stages (Figure 6). At first, the virus binds to host receptors (attachment); then it enters the host cells (penetration) via the mechanism of membrane fusion or endocytosis, after which, it releases its contents inside the host cells. The viral RNA translocates into the nucleus for replication and translate to viral proteins (biosynthesis). The new viral particles mature and are released into circulation (Figure 6).

As discussed earlier, coronaviruses are comprised of four structural proteins: spike (S), membrane (M), envelope (E), and nucleocapsid (N). ${ }^{51}$ The spike protein consists of a transmembrane trimetric glycoprotein that extends from the viral surface and defines the coronavirus host tropism and diversity. It has 2 functional subunits, the S1 subunit used for attaching to the host cell receptor, and the S2 subunit responsible for viral and cellular membrane fusion. Functional and structural investigation has revealed that the spike for SARS coronavirus-2 also bind to ACE2. ${ }^{52-54}$

ACE-2 has been identified as an active receptor for SARS$\mathrm{CoV}$ and is expressed highly in the lung, heart, ileum, kidney, and bladder. ${ }^{55}$ ACE-2 is highly expressed on the epithelial cells of the lung. After attaching to the host protein, the spike protein of SARS-CoV-2 undergoes protease cleavage ${ }^{56}$ at the S1/S2 cleavage site, making the S1 and S2 subunits remain non-covalently bound. The distal S1 subunit is involved in the balance of the membrane-anchored S2 subunit before fusion occurs. Subsequently, the cleavage of the S2 site is presumed to activate the spike protein for membrane fusion through irreversible conformational changes. A unique characteristic of the coronavirus spike is that it can be activated and cleave by a range of different proteases. ${ }^{57}$ Similarly, ubiquitous expression and the presence of the furin cleavage site (RPPA sequence) at the S1/S2 site makes the SARS-CoV-2

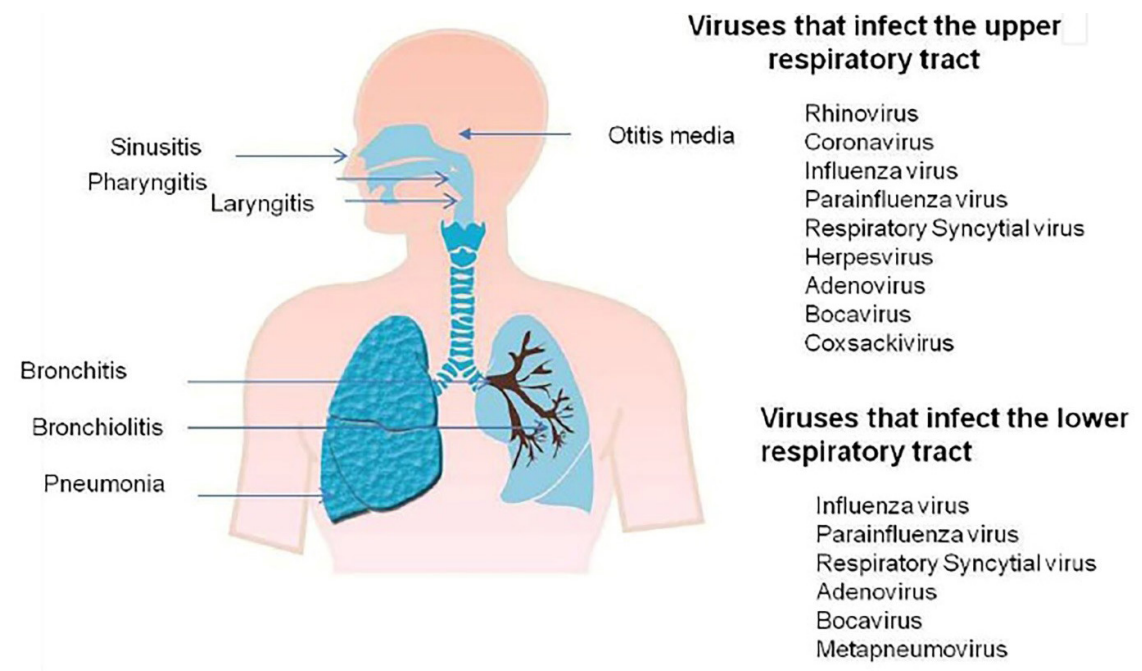

Figure 4. Main Syndromes of Viral Infection in the Respiratory Tract. ${ }^{44}$ 


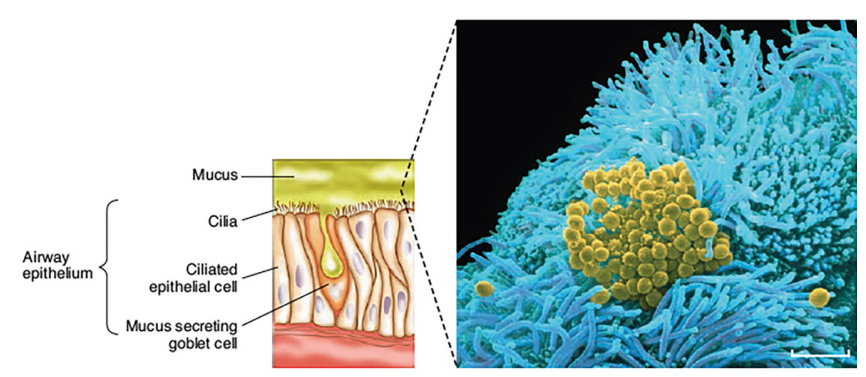

Figure 5. Defenses of Airway Epithelium. ${ }^{48}$

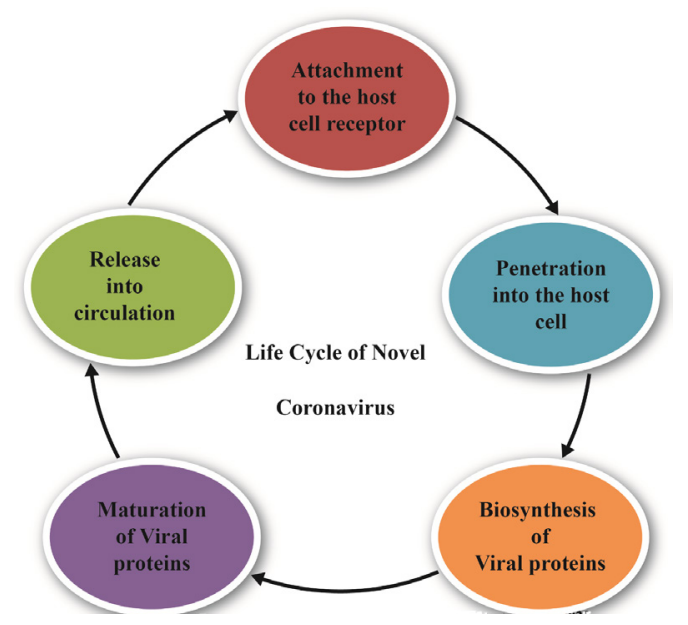

Figure 6. Life Cycle of COVID-19 in the Host Cell. ${ }^{50}$

unique and perhaps very pathogenic compared with other coronaviruses. During biosynthesis, however, the S1/S2 site of COVID-19 is totally subjected to cleavage in comparison with the COVID-19 spike, which is integrated into assembly without been cleaved.

These sites (S1/S2) are to be cleaved by other proteases, too, for example cathepsin $\mathrm{L}$ and transmembrane protease serine

\section{2 (TMPRSS2). ${ }^{56,58}$}

Through the renin-angiotensin system (RAS), the inhibition of ACE-2 promotes lung injury. ${ }^{60}$ In the pulmonary RAS, ACE-2 converts angiotensin II, an octapeptide hormone, to angiotensin 1-7 which is a heptapeptide hormone (Figure 6). Angiotensin II hormone induces pulmonary inflammation and activates the tumor necrosis factor (TNF) signaling pathway and mitogen-activated protein kinase signaling pathway (MAPK) to enhance lung injury. ${ }^{61,62}$ Angiotensin-1-7 prevents inflammation to protect the lungs from injury ${ }^{63}$ by hindering the MAPK signaling pathway, ${ }^{64}$ reducing cytokines release, ${ }^{65}$ and down-regulating RHOA (ras homolog family member A) pathway. ${ }^{66}$ Therefore, ACE-2 inhibition will increase the Angiotensin II level, decrease angiotensin-1-7, and promote dysregulation of different downstream pathways including MAPK and TNF signaling pathways to enhance lung injury (Figure 7). These pathways are deregulated in lung tissue from deceased COVID-19 patients. Based on this evidence, it was hypothesized that the inhibition of ACE-2 could possibly be the major molecular mechanism of lung injury in COVID-19. However, other pathways associated with cancers (for example proteoglycans in cancer and viral carcinogenesis) or cardiovascular diseases (for example viral myocarditis) reveal considerably enriched outcomes. These outcomes could help to clarify the increased risks of mortality among patients with COVID-19 with underlying health conditions (such as cancers or heart disease). ${ }^{9,67}$ Furthermore, myocarditis has been clinical examined in COVID-19 individuals, ${ }^{68}$ and a direct relation between the two maladies has been revealed.

\section{Pathogenesis of COVID-19 Infection in Organs}

In autopsy examination, pulmonary pathology was observed for both early and late phase ${ }^{69}$ SARS-CoV-2 individuals, the formation of mononuclear cells, macrophages infiltrating air spaces, hyaline membranes together with diffuse thickening of the alveolar wall and alveolar damage. Viral particles were

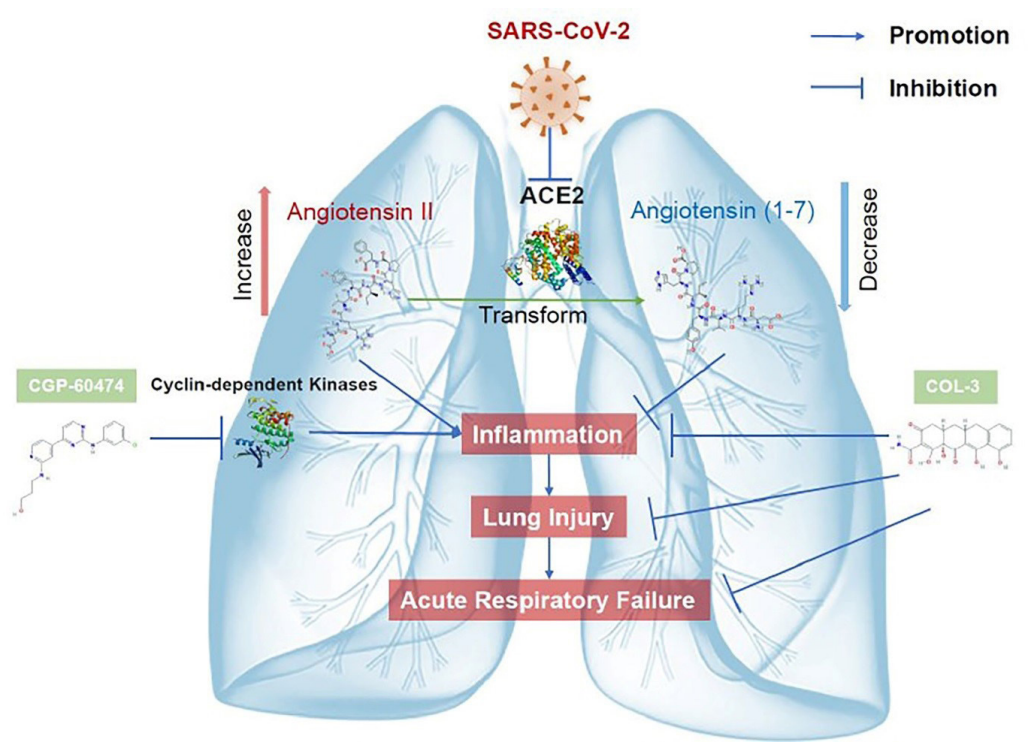

Figure 7. Proposed Mechanisms of Lung Injury by SARS-CoV-2. ${ }^{59}$ 


\section{Review Highlights}

\section{What Is Already Known?}

Coronaviruses are one of the largest known RNA with different genera $(\alpha-, \beta-, \gamma$-, and $\delta$-CoVs). The ongoing COVID-19 pandemic, which started in Wuhan, China in 2019, is an acute respiratory disease which can result in respiratory breakdown and possibly death. It is caused by the novel severe acute respiratory syndrome coronavirus 2 (SARS-CoV-2). The lung is the weakest internal organ, and it is prone to infection due to its continual contact with airborne particles, making lung infection/breakdown inevitable. Some infected individuals suffer nasal congestion; others may have no certain symptoms. Upper respiratory infections are a major type of infection suffered by humans, while infection of the lower respiratory tract took only a small percentage.

\section{What This Study Adds?}

It has recently been confirmed that the virus gains entrance into the host cell by binding to the host cell receptor and penetrating via the endocytosis. Upon entry, the virus releases its contents inside the host cell and moves into the nucleus where replication takes place. Afterwards, new viral particles are released into circulation for another phase of infection. The high expression of ACE-2, which has been identified as an active receptor for SARS-CoV in the lungs, makes the respiratory airways more susceptible to viral invasion. Other organs like the heart, kidney, bladder, and ileum have been shown to have high ACE2 expression, causing individuals with underlying health conditions or organ dysfunction to be more prone to the virus attack and less likely to survive it.

detected in type 2 alveolar epithelial cells and the bronchial by electron microscopy. Also, hilar lymph node necrosis, edema, scattered degeneration of neurons in the brain, focal hemorrhage in the kidney, spleen atrophy, and enlarged liver with inflammatory cell infiltration were observed in some individuals. $^{70,71}$

The viral particles of COVID-19 have also been isolated from the respiratory, fecal, and urine samples ${ }^{71}$ of COVID-19 patients, indicating multiple organ failure in individuals with severe COVID-19 caused partially by direct attack from the virus. To date, there has been no report concerning post-mortem investigations on the spread of viral particles through autopsy. Apart from the lungs, whether SARS-CoV-2 can directly attack organs with high ACE-2 expression and possibly with alternative cell receptors for SARS coronavirus-2 has to be further studied. ${ }^{71}$

\section{Conclusion}

The pandemic caused by the novel coronavirus is a life issue with global effects. With a lack of basic therapeutic interventions, existing management is aimed majorly at reducing the spread and transmission of the virus and providing supportive and palliative care for affected people.
This review helps deliver insight into the effects of COVID-19 on the pathogenesis of respiratory disease and its proposed mechanism of action in lung injury. Coronavirus can be spread from human to human through close contact and airborne droplets generated via coughing, sneezing, and kissing. Therefore, avoiding these activities with infected partners, friends, and relatives is of vital importance.

\section{Authors' Contributions}

All authors contributed in all parts of the study and approved the final copy of this article.

\section{Conflict of Interest Disclosures}

The authors declare no conflicts of interest regarding this work.

\section{Ethical Approval}

Not applicable.

\section{Funding/Support}

None.

\section{Acknowledgments}

The authors express their utmost respect to Professor Oluwatosin Adekunle Adaramoye of Drug Metabolism and Toxicology Laboratories, Department of Biochemistry, University of Ibadan, Ibadan, Nigeria.

\section{References}

1. Yin $Y$, Wunderink RG. MERS, SARS and other coronaviruses as causes of pneumonia. Respirology. 2018;23(2):130-137. doi:10.1111/resp. 13196

2. Lamarre A, Talbot PJ. Effect of $\mathrm{pH}$ and temperature on the infectivity of human coronavirus 229E. Can J Microbiol. 1989;35(10):972974. doi:10.1139/m89-160.

3. Global, regional, and national life expectancy, all-cause mortality, and cause-specific mortality for 249 causes of death, 1980-2015: a systematic analysis for the Global Burden of Disease Study 2015. Lancet. 2016;388(10053):1459-1544. doi:10.1016/s01406736(16)31012-1.

4. World Health Organization (WHO). Global Status Report on Noncommunicable Diseases. Geneva: WHO; 2014.

5. Yin Y, Wunderink RG. MERS, SARS and other coronaviruses as causes of pneumonia. Respirology. 2018;23(2):130-137. doi:10.1111/resp.13196.

6. Chen Y, Liu Q, Guo D. Emerging coronaviruses: Genome structure, replication, and pathogenesis. J Med Virol. 2020;92(4):418-423. doi:10.1002/jmv.25681

7. van der Hoek L, Pyrc K, Jebbink MF, et al. Identification of a new human coronavirus. Nat Med. 2004;10(4):368-373. doi:10.1038/ nm1024.

8. Woo PC, Lau SK, Chu CM, et al. Characterization and complete genome sequence of a novel coronavirus, coronavirus HKU1, from patients with pneumonia. J Virol. 2005;79(2):884-895. doi:10.1128/jvi.79.2.884-895.2005.

9. Li Q, Guan X, Wu P, et al. Early transmission dynamics in Wuhan, China, of novel coronavirus-infected pneumonia. N Engl J Med. 2020;382(13):1199-1207. doi:10.1056/NEJMoa2001316.

10. Zhu N, Zhang D, Wang W, et al. A novel coronavirus from patients with pneumonia in China, 2019. N Engl J Med. 2020;382(8):727733. doi:10.1056/NEJMoa2001017.

11. Chan JF, Yuan S, Kok KH, et al. A familial cluster of pneumonia associated with the 2019 novel coronavirus indicating person- 
to-person transmission: a study of a family cluster. Lancet. 2020;395(10223):514-523. doi:10.1016/s0140-6736(20)301549.

12. Phan LT, Nguyen TV, Luong QC, et al. Importation and human-tohuman transmission of a novel coronavirus in Vietnam. $\mathrm{N}$ Engl J Med. 2020;382(9):872-874. doi:10.1056/NEJMc2001272.

13. Rothe C, Schunk M, Sothmann P, et al. Transmission of 2019-nCoV infection from an asymptomatic contact in Germany. N Engl J Med. 2020;382(10):970-971. doi:10.1056/NEJMc2001468.

14. Holshue ML, DeBolt C, Lindquist S, et al. First case of 2019 novel coronavirus in the United States. N Engl J Med. 2020;382(10):929936. doi:10.1056/NEJMoa2001191.

15. Goldsmith CS, Tatti KM, Ksiazek TG, et al. Ultrastructural characterization of SARS coronavirus. Emerg Infect Dis. 2004;10(2):320-326. doi:10.3201/eid1002.030913.

16. Wan Y, Shang J, Graham R, Baric RS, Li F. Receptor recognition by the novel coronavirus from Wuhan: an analysis based on decadelong structural studies of SARS coronavirus. J Virol. 2020;94(7). doi:10.1128/jvi.00127-20.

17. Vicenzi E, Canducci F, Pinna D, et al. Coronaviridae and SARS-associated coronavirus strain HSR1. Emerg Infect Dis. 2004;10(3):413-418. doi:10.3201/eid1003.030683.

18. Gosert R, Kanjanahaluethai A, Egger D, Bienz K, Baker SC. RNA replication of mouse hepatitis virus takes place at doublemembrane vesicles. J Virol. 2002;76(8):3697-3708. doi:10.1128/ jvi.76.8.3697-3708.2002.

19. Song $Z, X u Y, B a o ~ L$, et al. From SARS to MERS, thrusting coronaviruses into the spotlight. Viruses. 2019;11(1). doi:10.3390/ v11010059.

20. Holmes KV, Enjuanes L. Virology. The SARS coronavirus: a postgenomic era. Science. 2003;300(5624):1377-1378. doi:10.1126/science.1086418.

21. Du L, He Y, Zhou Y, Liu S, Zheng BJ, Jiang S. The spike protein of SARS-CoV--a target for vaccine and therapeutic development. Nat Rev Microbiol. 2009;7(3):226-236. doi:10.1038/nrmicro2090.

22. Ruan YJ, Wei $C L$, Ee AL, et al. Comparative full-length genome sequence analysis of 14 SARS coronavirus isolates and common mutations associated with putative origins of infection. Lancet. 2003;361(9371):1779-1785. doi:10.1016/s0140-6736(03)134149

23. Marra MA, Jones SJ, Astell CR, et al. The Genome sequence of the SARS-associated coronavirus. Science. 2003;300(5624):13991404. doi:10.1126/science.1085953.

24. Letko M, Marzi A, Munster V. Functional assessment of cell entry and receptor usage for SARS-CoV-2 and other lineage B betacoronaviruses. Nat Microbiol. 2020;5(4):562-569. doi:10.1038/s41564-020-0688-y.

25. Lei J, Kusov Y, Hilgenfeld R. Nsp3 of coronaviruses: structures and functions of a large multi-domain protein. Antiviral Res. 2018;149:58-74. doi:10.1016/j.antiviral.2017.11.001.

26. Cascella M, Rajnik M, Cuomo A, Dulebohn SC, Di Napoli R. Features, Evaluation and Treatment Coronavirus (COVID-19). Treasure Island, FL: StatPearls Publishing; 2020.

27. Pyle CJ, Uwadiae FI, Swieboda DP, Harker JA. Early IL-6 signalling promotes IL-27 dependent maturation of regulatory T cells in the lungs and resolution of viral immunopathology. PLoS Pathog. 2017;13(9):e1006640. doi:10.1371/journal.ppat.1006640.

28. Bennardo F, Buffone C, Giudice A. New therapeutic opportunities for COVID-19 patients with Tocilizumab: possible correlation of interleukin-6 receptor inhibitors with osteonecrosis of the jaws. Oral Oncol. 2020;106:104659. doi:10.1016/j. oraloncology.2020.104659.

29. Chen Y, Liu Q, Guo D. Emerging coronaviruses: Genome structure, replication, and pathogenesis. J Med Virol. 2020;92(4):418-423. doi:10.1002/jmv.25681.

30. Chen $\mathrm{C}$, Zhang XR, Ju ZY, He WF. [Advances in the research of mechanism and related immunotherapy on the cytokine storm induced by coronavirus disease 2019]. Zhonghua
Shao Shang Za Zhi. 2020;36(6):471-475. doi:10.3760/ cma.j.cn501120-20200224-00088.

31. Clarivate Analytics Solution. Disease Briefing: Coronaviruses. Clarivate Analytics Solution; 2020.

32. Rautiainen $\mathrm{M}$, Nuutinen J, Kiukaanniemi H, Collan Y. Ultrastructural changes in human nasal cilia caused by the common cold and recovery of ciliated epithelium. Ann Otol Rhinol Laryngol. 1992;101(12):982-987. doi:10.1177/000348949210101204.

33. Johnston SL, Pattemore PK, Sanderson G, et al. Community study of role of viral infections in exacerbations of asthma in 9-11 year old children. BMJ. 1995;310(6989):1225-1229. doi:10.1136/ bmj.310.6989.1225.

34. Preliminary estimates of the prevalence of selected underlying health conditions among patients with coronavirus disease 2019-United States, February 12-March 28, 2020. MMWR Morb Mortal Wkly Rep. 2020;69(13):382-386. doi:10.15585/mmwr. mm6913e2.

35. Su N, Lin J, Chen P, et al. Evaluation of asthma control and patient's perception of asthma: findings and analysis of a nationwide questionnaire-based survey in China. J Asthma. 2013;50(8):861870. doi:10.3109/02770903.2013.808346.

36. Bosch BJ, van der Zee R, de Haan CA, Rottier PJ. The coronavirus spike protein is a class I virus fusion protein: structural and functional characterization of the fusion core complex. J Virol. 2003;77(16):8801-8811. doi:10.1128/jvi.77.16.8801-8811.2003.

37. Yamaya $M$, Nishimura $H$, Deng $X$, et al. Inhibitory effects of glycopyrronium, formoterol, and budesonide on coronavirus HCoV-229E replication and cytokine production by primary cultures of human nasal and tracheal epithelial cells. Respir Investig. 2020;58(3):155-168. doi:10.1016/j.resinv.2019.12.005.

38. Matsuyama S, Kawase M, Nao N, et al. The inhaled corticosteroid ciclesonide blocks coronavirus RNA replication by targeting viral NSP15. bioRxiv. 2020. doi:10.1101/2020.03.11.987016.

39. Iwabuchi K, Yoshie K, Kurakami Y, Takahashi K, Kato Y, Morishima T. Therapeutic potential of ciclesonide inahalation for COVID-19 pneumonia: report of three cases. J Infect Chemother. 2020;26(6):625-632. doi:10.1016/j.jiac.2020.04.007.

40. Stockman LJ, Bellamy R, Garner P. SARS: systematic review of treatment effects. PLoS Med. 2006;3(9):e343. doi:10.1371/ journal.pmed.0030343.

41. Flint SJ, Enquist LW, Krug RM, Rocaniello VR, Skalka AM. Principles of Virology: Molecular Biology, Pathogenesis, and Control. Washington, DC: ASM Press; 2000.

42. Gottwein E, Cullen BR. Viral and cellular microRNAs as determinants of viral pathogenesis and immunity. Cell Host Microbe. 2008;3(6):375-387. doi:10.1016/j.chom.2008.05.002.

43. Ghosh Z, Mallick B, Chakrabarti J. Cellular versus viral microRNAs in host-virus interaction. Nucleic Acids Res. 2009;37(4):10351048. doi:10.1093/nar/gkn1004.

44. Manjarrez ME, Rosete D, Higuera A, Ocádiz-Delgado R, PérezPadilla JR, Cabello C. Start of a pandemic: influenza A H1N1 virus. In: Chanei M, ed. Respiratory Diseases. Rieja, Croatia: InTech Open Access Publisher; 2012:217-242. doi:10.13140/2.1.3340.2564.

45. Corjon S, Gonzalez G, Henning P, et al. Cell entry and trafficking of human adenovirus bound to blood factor $\mathrm{X}$ is determined by the fiber serotype and not hexon:heparan sulfate interaction. PLoS One. 2011;6(5):e18205. doi:10.1371/journal.pone.0018205.

46. López MJ, Manjarrez ME, Zavala TJ. Microbiología. Bacteriología y Virología. México: Méndez editors; 2010:912.

47. Dolin R, Wright PF. Viral Infections of the Respiratory Tract. New York: Marcel Dekker; 1999:432.

48. Infectious Disease Affecting the Respiratory System- chapter 20. Jones and Bartlett; 2003:676.

49. The Novel Coronavirus Pneumonia Emergency Response Epidemiology Team. The epidemiological characteristics of an outbreak of 2019 novel coronavirus diseases (COVID19)—China, 2020. China CDC Weekly. 2020;2(8):113-122. doi:10.46234/ ccdcw2020.032. 
50. Chen Y, Guo Y, Pan Y, Zhao ZJ. Structure analysis of the receptor binding of 2019-nCoV. Biochem Biophys Res Commun. 2020;525(1):135-140. doi:10.1016/j.bbrc.2020.02.071.

51. Walls AC, Park YJ, Tortorici MA, Wall A, McGuire AT, Veesler D. Structure, function, and antigenicity of the SARS-CoV-2 spike glycoprotein. Cell. 2020;181(2):281-292.e286. doi:10.1016/j. cell.2020.02.058

52. Letko M, Marzi A, Munster V. Functional assessment of cell entry and receptor usage for SARS-CoV-2 and other lineage B betacoronaviruses. Nat Microbiol. 2020;5(4):562-569. doi:10.1038/s41564-020-0688-y.

53. Zou X, Chen K, Zou J, Han P, Hao J, Han Z. Single-cell RNA-seq data analysis on the receptor ACE2 expression reveals the potential risk of different human organs vulnerable to 2019-nCoV infection. Front Med. 2020;14(2):185-192. doi:10.1007/s11684-020-07540 .

54. Ou X, Liu Y, Lei X, et al. Characterization of spike glycoprotein of SARS-CoV-2 on virus entry and its immune cross-reactivity with SARS-CoV. Nat Commun. 2020;11(1):1620. doi:10.1038/s41467020-15562-9.

55. Belouzard S, Millet JK, Licitra BN, Whittaker GR. Mechanisms of coronavirus cell entry mediated by the viral spike protein. Viruses. 2012;4(6):1011-1033. doi:10.3390/v4061011.

56. Hoffmann M, Kleine-Weber $\mathrm{H}$, Schroeder S, et al. SARS-CoV-2 cell entry depends on ACE2 and TMPRSS2 and is blocked by a clinically proven protease inhibitor. Cell. 2020;181(2):271-280. e278. doi:10.1016/j.cell.2020.02.052.

57. Kuba K, Imai Y, Penninger JM. Angiotensin-converting enzyme 2 in lung diseases. Curr Opin Pharmacol. 2006;6(3):271-276. doi:10.1016/j.coph.2006.03.001.

58. Marshall RP, Gohlke P, Chambers RC, et al. Angiotensin II and the fibroproliferative response to acute lung injury. Am J Physiol Lung Cell Mol Physiol. 2004;286(1):L156-164. doi:10.1152/ ajplung.00313.2002.

59. Tao W, Li PS, Xu G, et al. Soluble epoxide hydrolase plays a vital role in angiotensin ii-induced lung injury in mice. Shock. 2018;50(5):589-594. doi:10.1097/shk.0000000000001067.

60. Lu W, Kang J, Hu K, et al. Angiotensin-(1-7) inhibits inflammation and oxidative stress to relieve lung injury induced by chronic intermittent hypoxia in rats. Braz J Med Biol Res. 2016;49(10):e5431. doi:10.1590/1414-431×20165431.

61. Meng Y, Yu CH, Li W, et al. Angiotensin-converting enzyme 2/ angiotensin-(1-7)/Mas axis protects against lung fibrosis by inhibiting the MAPK/NF-kB pathway. Am J Respir Cell Mol Biol. 2014;50(4):723-736. doi:10.1165/rcmb.2012-0451OC.

62. Rodrigues Prestes TR, Rocha NP, Miranda AS, Teixeira AL, Simoes ESAC. The anti-inflammatory potential of ACE2/angiotensin-(1-7)/ Mas receptor axis: evidence from basic and clinical research. Curr Drug Targets. 2017;18(11):1301-1313. doi:10.2174/1389450117 666160727142401.

63. Meng Y, Li T, Zhou GS, et al. The angiotensin-converting enzyme 2/angiotensin (1-7)/Mas axis protects against lung fibroblast migration and lung fibrosis by inhibiting the NOX4-derived ROSmediated RhoA/Rho kinase pathway. Antioxid Redox Signal. 2015;22(3):241-258. doi:10.1089/ars.2013.5818.

64. [The epidemiological characteristics of an outbreak of 2019 novel coronavirus diseases (COVID-19) in China]. Zhonghua Liu Xing Bing Xue Za Zhi. 2020;41(2):145-151. doi:10.3760/cma.j.is sn.0254-6450.2020.02.003.

65. Inciardi RM, Lupi L, Zaccone G, et al. Cardiac involvement in a patient with coronavirus disease 2019 (COVID-19). JAMA Cardiol. 2020;5(7):1-6. doi:10.1001/jamacardio.2020.1096.

66. He B, Garmire L. Prediction of Repurposed Drugs for Treating Lung Injury in COVID-19. 2020 Mar 30: arXiv:2003.14333v2.

67. Xu Z, Shi L, Wang Y, et al. Pathological findings of COVID-19 associated with acute respiratory distress syndrome. Lancet Respir Med. 2020;8(4):420-422. doi:10.1016/s2213-2600(20)30076-x.

68. National Health Commission. Diagnosis and treatment protocol for novel coronavirus pneumonia (Trial Version 7). Chin Med J (Engl). 2020;133(9):1087-95.

69. Yao XH, Li TY, He ZC, et al. [A pathological report of three COVID-19 cases by minimal invasive autopsies]. Zhonghua Bing Li Xue Za Zhi. 2020;49(5):411-417. doi:10.3760/ cma.j.cn112151-20200312-00193.

70. Wang W, XuY, Gao R, et al. Detection of SARS-CoV-2 in different types of clinical specimens. JAMA. 2020;323(18):1843-1844. doi:10.1001/jama.2020.3786.

71. Yuki K, Fujiogi M, Koutsogiannaki S. COVID-19 pathophysiology: a review. Clin Immunol. 2020;215:108427. doi:10.1016/j. clim.2020.108427. 